\title{
Influência do Teor de Polipropileno Modificado com Anidrido Maleico nas Propriedades do Nanocompósito PP/EPDM/Argila Organofílica
}

\author{
Fernanda C. F. Braga \\ Programa de Pós-Graduação em Química, UERJ \\ Marcia G. Oliveira \\ Divisão de Processamento e Caracterização de Materiais, INT \\ Cristina R. G. Furtado \\ Departamento de Processos Químicos, UERJ
}

\begin{abstract}
Resumo: Neste trabalho foram obtidos nanocompósitos de elastômeros termoplásticos à base de PP/EPDM/argila organofílica pela técnica de intercalação no estado fundido em câmara interna de mistura. Foram preparados concentrados de PP-MA (agente interfacial) e Claytone 40 (argila organofílica) nas proporções de 1:1, 2:1 e 3:1 que posteriormente foram adicionados na matriz PP/EPDM, previamente preparada, mantendo o teor de argila fixo em $5 \%$ (em massa). Foram investigadas as propriedades mecânicas de módulo elástico, tração e alongamento na ruptura, reologia e morfologia, a fim de estabelecer a influência da razão PP-MA/argila organofílica no comportamento dos nanocompósitos. Os resultados mostraram que a adição de teores crescentes de PP-MA melhorou a dispersão da argila organofílica na matriz de PP/EPDM. Conforme revelado pela difração de raio X e confirmados pela reometria foram obtidos nanocompósitos com estruturas mistas intercaladas e esfoliadas, que resultaram em maiores valores de módulo e deformação na ruptura.
\end{abstract}

Palavras-chave: Nanocompósito, elastômero termoplástico, argila organofílica, PP-MA.

\section{Influence from the Concentration of Interfacial Agent on the Properties of PP/EPDM/Organoclay Nanocomposites}

\begin{abstract}
In this work, nanocomposites were prepared with PP/EPDM thermoplastic elastomer and organoclay by melt intercalation in an internal mixture chamber. Masterbatches composed of PP-MA/organoclay in proportions of 1:1, 2:1 and 3:1 were prepared and further added to a PP/EPDM thermoplastic elastomer, keeping the clay content fixed at 5\% (by weight). The tensile properties, rheology and the morphology (organoclay dispersion, crystalline structure and crystallinity degree) of nanocomposites were studied to establish the influence of the PP-MA/Organoclay ratio in the system. The results showed that the addition of increasing levels of PP-MA improved the dispersion of organoclay in the PP/EPDM matrix. As revealed in x-ray diffraction and confirmed by rheology measurements nanocomposites were obtained with mixed intercalated and exfoliated structures, resulting in higher tensile modulus and elongation at break.
\end{abstract}

Keywords: Nanocomposite, thermoplastic elastomers, organoclay, PP-MA.

\section{Introdução}

Elastômeros termoplásticos (TPE) à base de polipropileno (PP) e de terpolímero de etileno-propileno-dieno (EPDM) ganharam notoriedade pela facilidade de preparação de compósitos e pela combinação das altas deformações de um elastômero e do processamento de um termoplástico ${ }^{[1]}$. A adição de cargas em escala nanométrica a essas matrizes poliméricas, tem sido um atrativo potencial em função das características que a carga inorgânica pode apresentar quando bem dispersa no polímero orgânico. Esses materiais inorgânicos utilizados como nanocarga (geralmente silicatos em camadas), além de apresentarem elevada razão de aspecto, são suscetíveis a intercalação de macromoléculas e são os mais utilizados em função da fácil disponibilidade e tratamento de superfície ${ }^{[2]}$. A utilização de baixos teores de organo-argila $(\leq 5 \%)^{[3-5]}$ é essencial para aumentar a superfície de contato com o polímero, e conseqüentemente promover boa dispersão da mesma na matriz, minimizando o efeito de agregação das lamelas.

$\mathrm{Na}$ literatura já há alguns exemplos de nanocompósitos cuja matriz é a mistura PP/EPDM, predominantemente ricas em polipropileno e assumindo um comportamento típico de um plástico tenacificado. É senso comum entre os autores a necessidade do uso de um compatibilizante e o preferido é o polipropileno modificado com anidrido maleico. De fato, os nanocompósitos obtidos apresentaram morfologia intercalada, acompanhada de aumento de módulo e viscosidade no estado fundido ${ }^{[6]}$.

O agente compatibilizante, PP-MA, atua favorecendo a interação entre a matriz apolar, mistura PP/EPDM, e a organo-argila, porém a sua quantidade no sistema final altera a razão PP/EPDM e por conseqüência o comportamento mecânico e reológico do nanocompósito ${ }^{[7]}$. Assim, os autores destacam em suas conclusões a necessidade de otimizar a quantidade de PP-MA no sistema.

Assim, este trabalho visa complementar as informações da literatura no que tange à otimização da quantidade de PP-MA no nanocompósito PP/EPDM/organo-argila, com quantidades equivalentes de PP e EPDM. Para tanto foram preparados concentrados compostos por PP-MA e organo-argila em diferentes proporções, os quais foram posteriormente diluídos na mistura PP/ EPDM, também previamente preparada, mantendo-se o teor final de organo-argila no nanocompósito em 5\%. Os nanocompósitos obtidos foram avaliados em termos de morfologia, reologia, cristalinidade, propriedades térmicas e de tração.

Autor para correspondência: Fernanda C. F. Braga, Programa de Pós-graduação em Química, Universidade do Estado do Rio de Janeiro - UERJ,

Rua São Francisco Xavier, 524, Maracanã, 3o andar, CEP 20550-900, Rio de Janeiro, RJ, Brasil, e-mail: fc_braga@yahoo.com.br 


\section{Experimental}

\section{Materiais}

A matriz polimérica de elastômero termoplástico estudada foi composta de $50 \%$ de polipropileno (MFI = $11 \mathrm{~g} / 10 \mathrm{~min}$ ) da Suzano Petroquímica e 50\% de terpolímero de etileno-propileno-dieno (Keltan 21) da DSM Elastômeros (viscosidade mooney (ML) $1+4125^{\circ} \mathrm{C}=25 \pm 4 \mathrm{UM}$ ), buscando a obtenção de uma matriz com características mais próximas a de elastômero termoplástico. A argila empregada foi a montmorilonita comercial modificada organicamente com o cloreto de diestearil dimetil amônio, com concentração de 84 meq. $100 \mathrm{~g}^{-1}$, denominada Claytone $40 \mathrm{da}$ Bentonit União Nordeste (BUN). O agente interfacial utilizado foi o polipropileno modificado com anidrido maleico (PP-MA), Fusabond MD353D, da Du Pont com MFI = 22,4 g/10 min e teor de anidrido maleico igual a $1,4 \%$.

\section{Preparação dos nanocompósitos}

Todas as misturas, matriz de PP/EPDM (50:50), concentrados e nanocompósitos, foram preparadas por intercalação no estado fundido a $190{ }^{\circ} \mathrm{C}$ em câmara interna de mistura acoplada ao reômetro de torque, modelo Haake Polylab OS Rheodrive 4 utilizando rotores do tipo Cam, a $80 \mathrm{rpm}$. Os concentrados PP-MA/ Claytone 40 foram preparados nas proporções 1:1, 2:1 e 3:1, onde inicialmente, foi adicionado a câmara de mistura, o PP-MA por 3 minutos e posteriormente, a Claytone 40, totalizando 6 minutos de processamento. As condições das misturas foram determinadas a partir da estabilização do torque durante o processamento, e a quantidade de argila incorporada, foi definida em função dos resultados obtidos em estudos anteriores, ao variar-se os teores em 2,5; 5 e $7 \%$ em massa de argila organofílica.

Os nanocompósitos foram obtidos a partir da adição dos concentrados ao TPE (PP/EPDM), mantendo-se fixa a quantidade de argila no nanocompósito em 5\%. Ao TPE foi adicionado o concentrado por 3 minutos, perfazendo 5 minutos de mistura. Adotou-se como nomenclatura a sigla NC X:Y, onde: $\mathrm{NC}=$ nanocompósito e $\mathrm{X}: \mathrm{Y}=$ razão PP-MA/Claytone 40.

\section{Morfologia}

As micrografias foram realizadas em microscópio eletrônico de varredura FEI Company, modelo INSPECT S50, utilizando-se corpos de prova criofraturados e recobertos com carbono.

\section{Cristalinidade}

A caracterização estrutural dos concentrados e dos nanocompósitos foi avaliada por um difratômetro de raio X Rigaku, modelo Miniflex, à $25^{\circ} \mathrm{C}$ utilizando radiação $\operatorname{Cuk} \alpha(\lambda=0,1504 \mathrm{~nm})$, operando a $40 \mathrm{kV}$ e amperagem de $40 \mathrm{~mA}$ empregando uma taxa de varredura de $1^{\circ} \cdot \mathrm{m}^{-1}$. A distância interlamelar da argila no nanocompósito foi calculada utilizando a equação de Bragg $(\lambda=2 \mathrm{~d} \operatorname{sen} \theta)$ no intervalo de $2 \theta$ de 1 a $12^{\circ}$. A estrutura cristalina da fase PP também foi determinada por esta técnica no intervalo de $2 \theta$ de 10 a $40^{\circ}$.

\section{Propriedades térmicas}

O comportamento das temperaturas de fusão e de cristalização dos nanocompósitos foram avaliados por calorimetria diferencial de varredura (DSC) em equipamento TA Instruments, modelo DSC Q-100. As amostras foram submetidas ao aquecimento entre $25-200{ }^{\circ} \mathrm{C}$ em fluxo de $30 \mathrm{~mL} / \mathrm{min}$ de nitrogênio e taxa de aquecimento de $10{ }^{\circ} \mathrm{C} / \mathrm{min}$. Posteriormente, essas amostras foram resfriadas até $25^{\circ} \mathrm{C}$ e novamente aquecidas nas mesmas condições. Os valores de temperatura e entalpia de fusão cristalina foram retirados da curva do segundo ciclo de aquecimento.

\section{Propriedades reológicas}

As propriedades viscoelásticas dos nanocompósitos foram determinadas em um reômetro modular HAAKE MARS com geometria de placas paralelas (placas de $20 \mathrm{~mm}$ de diâmetro, distância entre as placas de $1 \mathrm{~mm}$ ). A análise de varredura de freqüência foi conduzida a temperatura de $200{ }^{\circ} \mathrm{C}$ na faixa de 0,1-100 rad/s, com amplitude de deformação de $1 \%$.

\section{Propriedades mecânicas}

Os corpos de prova para ensaio de tração foram obtidos segundo a norma DIN 53504 em injetora Battenfleld ${ }^{\mathrm{TM}}$, modelo Plus 35, $230{ }^{\circ} \mathrm{C}$ de temperatura de injeção, $9 \mathrm{MPa}$ de pressão de injeção e $20{ }^{\circ} \mathrm{C}$ de temperatura de molde. Os ensaios foram feitos em uma máquina universal de ensaios EMIC, modelo DL-2000, com célula de carga de $1 \mathrm{KN}$, velocidade de separação entre as garras de $50 \mathrm{~mm} / \mathrm{min}$, Os resultados foram obtidos a partir da média de sete corpos de prova.

\section{Resultados e Discussão}

\section{Grau de dispersão da Claytone 40}

Pela técnica de difração de raio X não foi possível detectar o pico característico da argila montmorilonita modificada nos concentrados (Figura 1a), tendo sido apresentados com características alargadas e de baixíssima intensidade. Desta forma, é possível esperar que os concentrados possuam uma estrutura mista, ou seja, podendo coexistir estruturas intercaladas e esfoliadas ${ }^{[8,9]}$.

Muito embora tenham sido obtidas estruturas esfoliadas com os concentrados PP-MA/Argila, detectou-se que a adição destes à matriz de TPE, promoveu a formação de estruturas parcialmente intercaladas, como ilustrado na Figura 1b. O aumento da viscosidade do sistema e o tamanho da cadeia polimérica possivelmente contribuíram para a mudança de estrutura, assim como a redução da polaridade do sistema ao inserir o concentrado em cadeia apolares de TPE. Essa observação foi feita a partir do deslocamento dos picos para menores valores de $2 \theta$ quando comparados a argila pura, além da redução da intensidade do pico de difração juntamente com o alargamento do mesmo. Quanto ao sistema TPE/argila organofílica, foi verificado que o pico de difração apresentou-se de forma mais intensa e definida. A ausência de moléculas polares na matriz de TPE não favoreceu a interação efetiva com a superfície polar da argila, indicando a limitada capacidade de intercalação dessa argila na ausência de um agente interfacial.

Para verificar a influência do agente interfacial na dispersão da argila foi realizada a microscopia eletrônica de varredura. $\mathrm{Na}$ Figura 2a-d é possível verificar a presença de pequenos domínios deformados, os quais podem ser a fase elastomérica (Figura 2c), cuja superfície de fratura é mais regular frente às demais amostras, onde se observa a redução do tamanho desses domínios. O contraste entre as fases PP e EPDM é reduzido na detecção dos elétrons secundários, não permitindo o aprofundamento de detalhes da morfologia destas fases. Segundo a literatura a presença da argila com um agente interfacial resulta na redução de tamanho e nos formatos irregulares dos domínios do elastômero, em função de efeitos reológicos e de barreira durante o processamento no estado fundido ${ }^{[7]}$. Não é possível visualizar nessas micrografias a existência 


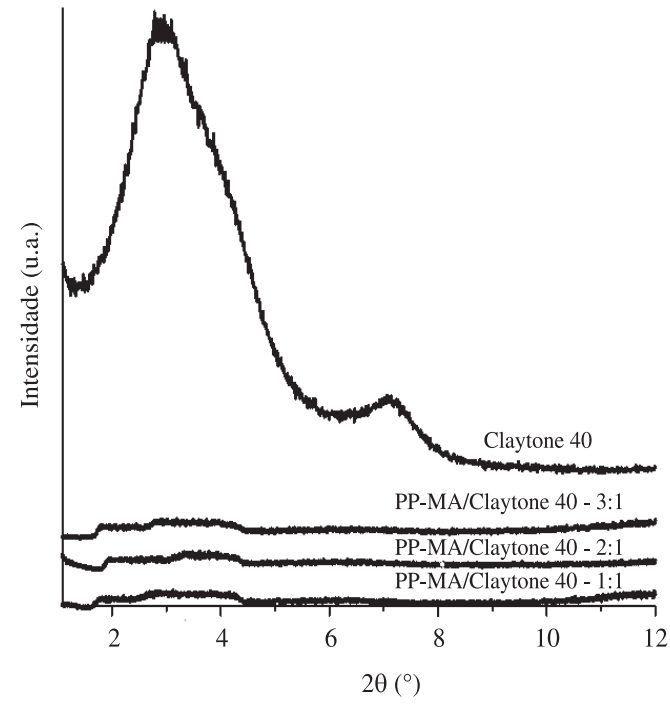

(a)

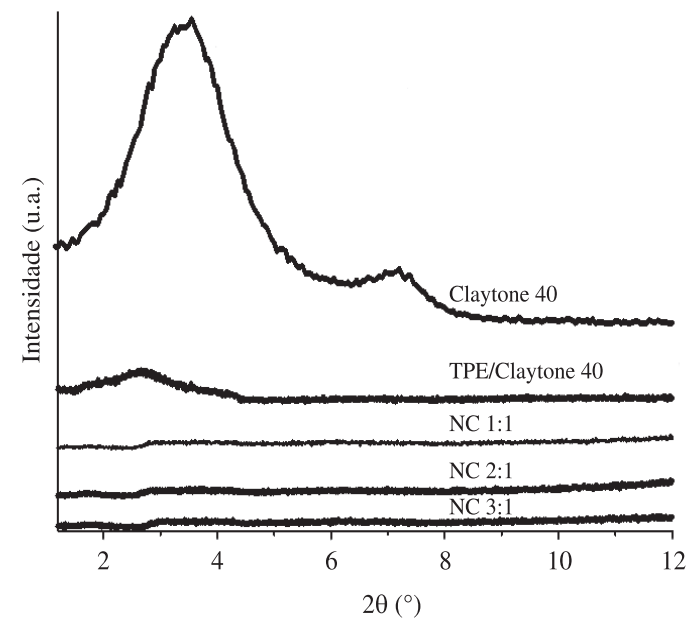

(b)

Figura 1. Difratogramas de raio X: a) masterbatch e b) nanocompósitos.

Tabela 1. Propriedades térmicas e grau de cristalinidade obtido da deconvolução dos picos cristalinos e halo amorfo do PP, TPE e nanocompósitos.

\begin{tabular}{lccc}
\hline \multirow{2}{*}{ Amostra } & DRX & \multicolumn{3}{c}{ DSC } \\
\cline { 2 - 4 } & $\mathbf{X}_{\mathbf{c}}$ & $\mathbf{T}_{\mathbf{c}}$ & $\mathbf{T}_{\mathbf{m}}$ \\
& {$[\mathbf{\%}]$} & {$\left[{ }^{\circ} \mathbf{C}\right]$} & {$\left[{ }^{\circ} \mathbf{C}\right]$} \\
\hline PP & 49,9 & 109 & 165 \\
TPE & 41,6 & 111 & 159 \\
TPE/Claytone 40 & 31,3 & 114 & 159 \\
NC 1:1 & 30,3 & 112 & 159 \\
NC 2:1 & 26,5 & 111 & 157 \\
NC 3:1 & 41,2 & 113 & 159 \\
\hline
\end{tabular}

de aglomerados de argila, porque possivelmente a topografia resultante da criofratura pode estar dificultando a observação das amostras. As regiões mais brilhantes observadas são reflexos do carregamento da amostra durante a análise. Para verificar o grau de distribuição e dispersão da argila na matriz de TPE, optou-se pela realização do mapeamento do elemento silício utilizando-se o detector EDS, tendo em vista que a argila é um aluminossilicato. A Figura 2e-h mostra os mapas, onde é possível observar a grande semelhança entre os nanocompósitos obtidos a partir da diluição dos concentrados, a exemplo do que já fora observado nas análises de DRX. Em contrapartida, o nanocompósito sem o PP-MA, Figura 2 a,e apresenta a formação de um grande aglomerado e uma dispersão desigual na região mapeada, reforçando assim a importância da presença do PP-MA na dispersão da Claytone 40 na matriz de TPE.

\section{Estrutura cristalina}

Os difratogramas foram obtidos na faixa de $2 \theta$ entre $10^{\circ}$ e $40^{\circ} \mathrm{e}$ foi possível constatar que a estrutura cristalina do PP é equivalente ao cristal tipo $\alpha$.

A adição da argila Claytone 40 resultou na redução do grau de cristalinidade $\left(\mathrm{X}_{c}\right)$ em relação ao TPE (mistura PP/EPDM 50:50). Este comportamento se manteve com a adição dos concentrados PP-MA/Claytone 40 nas razões de 1:1 e 2:1). Já a adição do concentrado na razão 3:1 (NC 3:1) promoveu alterações na estrutura cristalina da fase PP, sendo observado mais um pico no difratograma característico de uma possível forma $\gamma\left(2 \theta=17,21^{\circ}\right)$, como observado na Figura 3. Acredita-se que este comportamento seja decorrência do maior teor de PP-MA presente no sistema, possibilitando uma maior mobilidade da cadeia polimérica em função de sua baixa viscosidade.

Os resultados obtidos a partir das curvas de DSC apresentados na Tabela 1 assinalam que a adição da argila Claytone 40 com ou sem agente interfacial ocasionou um discreto aumento da temperatura de cristalização $\left(\mathrm{T}_{c}\right)$. Este comportamento combina com alguns relatos da literatura que destacam a ação nucleante da argila. No tocante à temperatura de fusão cristalina $\left(\mathrm{T}_{\mathrm{m}}\right)$ não foi observada modificação significativa em relação à matriz de TPE.

\section{Comportamento reológico}

A Figura 4 ilustra a variação da viscosidade complexa $\left(\eta^{*}\right)$ e do módulo elástico (G') em função da freqüência angular $(\omega)$ para as diferentes razões PP-MA/Argila adicionadas à na matriz de TPE. A adição da argila organofílica sem o agente interfacial (PP-MA) resultou em aumento dos valores de viscosidade em relação ao TPE, conforme comportamento característico da adição de cargas inorgânicas a uma matriz polimérica. Comportamento semelhante foi verificado para os nanocompósitos contendo PPMA, todavia, a viscosidade deles foi maior do que a viscosidade não só do TPE puro como também do TPE contendo apenas a argila organofílica, o que sugere a ação do PP-MA na modificação da estrutura do nanocompósito, seja ela intercalada ou esfoliada. Lembrando que o PP-MA possui uma viscosidade bem menor do que o PP e o EPDM, que formam a matriz de TPE, portanto seria natural que fosse observada uma redução da viscosidade, o que de fato não aconteceu, reforçando a hipótese do PP-MA agir efetivamente auxiliando a formação de estruturas intercaladas e/ ou esfoliadas. Alguns autores atribuem o aumento da viscosidade dos nanocompósitos, principalmente na região de baixa freqüência (0,1-1,0 rad/s), ao grau de esfoliação da argila organofílica na matriz polimérica $^{[7,9,10,11]}$. Por vezes o platô newtoniano é suprimido e a faixa de pseudoplasticidade é antecipada, assim como observado na Figura 4.

No tocante ao módulo elástico (G') observou-se valores maiores para os nanocompósitos contendo PP-MA, independente da razão PP-MA/argila, especialmente na região de baixa frequiência (0,1-1 rad/s). Notou-se ainda que a inclinação da curva nessa faixa de freqüência é diferente para os nanocompósitos quando comparados ao TPE e ao TPE contendo apenas argila organofílica. De fato, esta menor inclinação remete a um comportamento reológico 


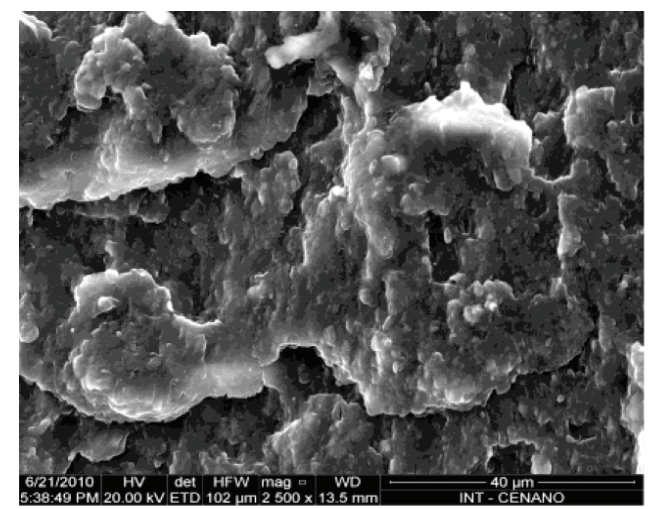

(a)

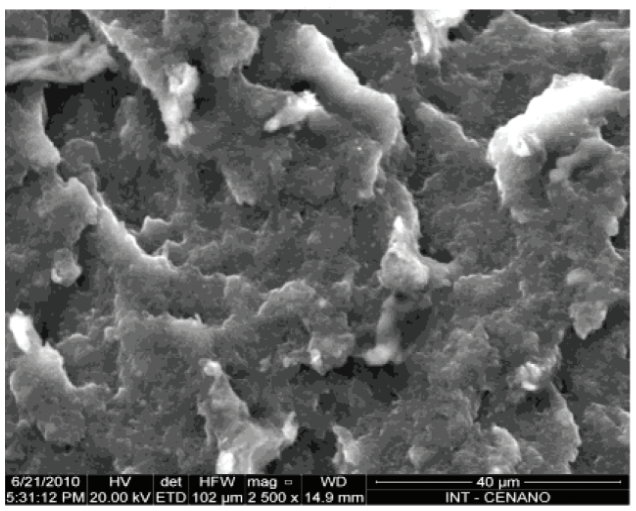

(b)

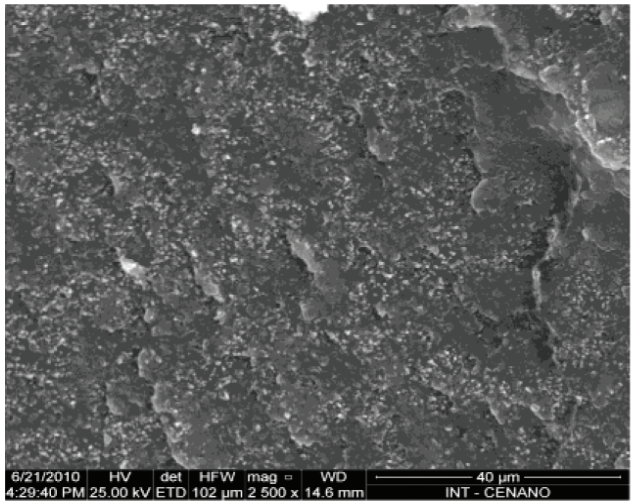

(c)

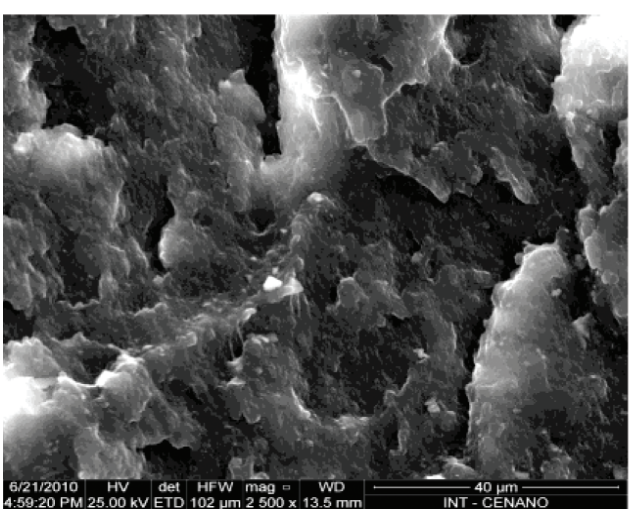

(d)

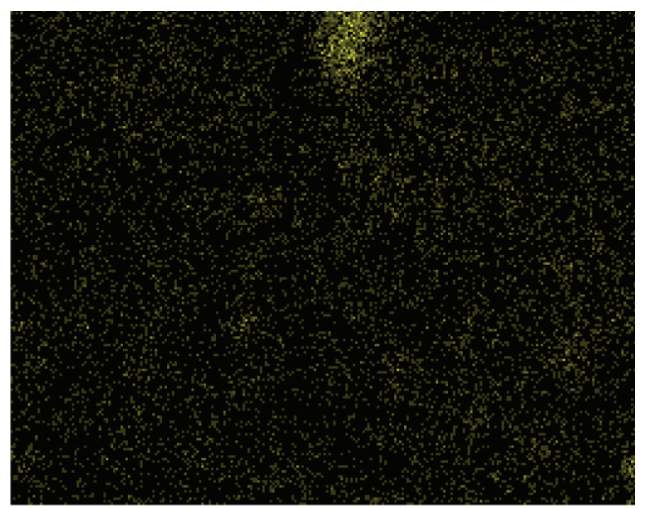

(e)

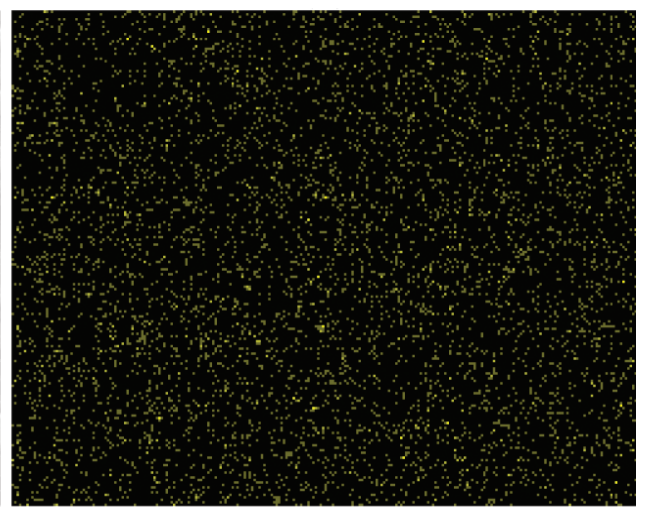

(f)

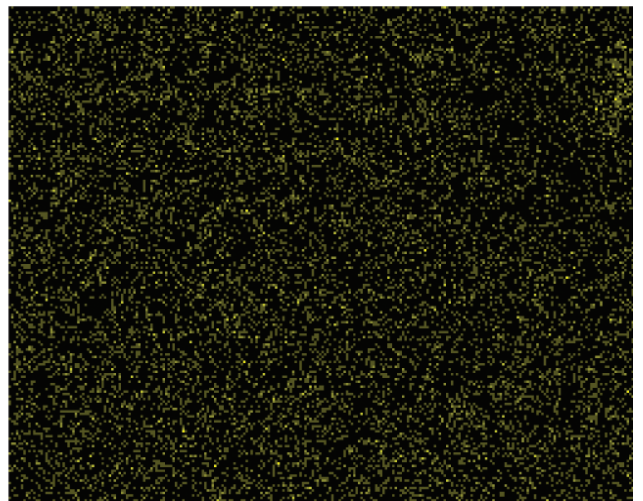

(g)

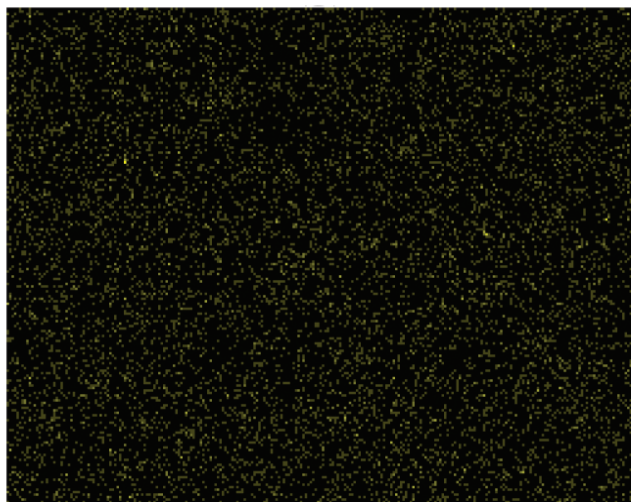

(h)

Figura 2. Micrografias dos nanocompósitos com teores crescentes de PP-MA. Com detector ES: a) TPE/Claytone 40; b) NC 1:1; c) NC 2:1 e d) NC 3:1; com detector EDS para mapeamento de Si: e) TPE/Claytone 40; f) NC 1:1; g) NC 2:1 e h) NC 3:1. 


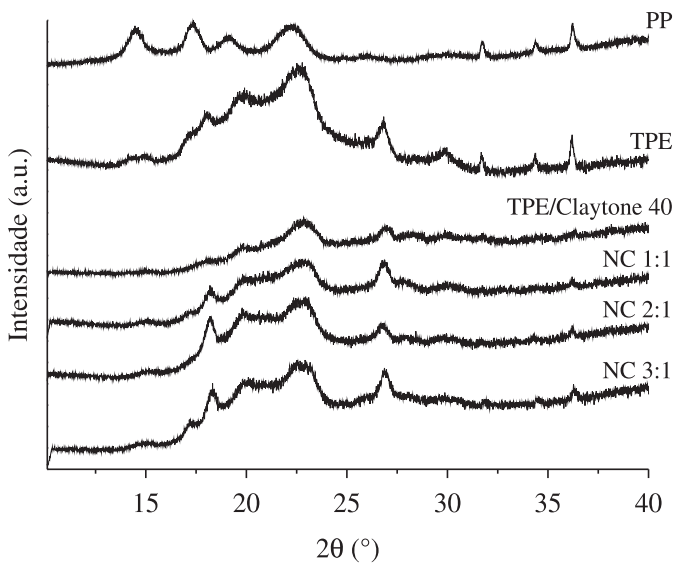

Figura 3. Difratogramas das amostras de PP, TPE e nanocompósitos.

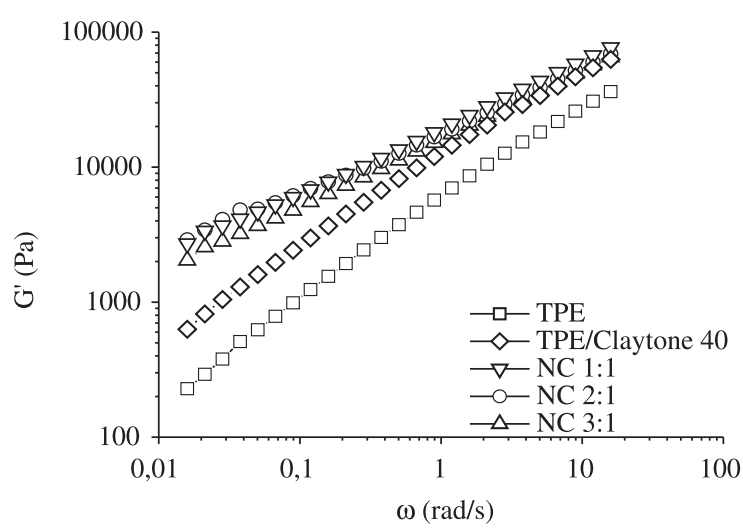

(a)

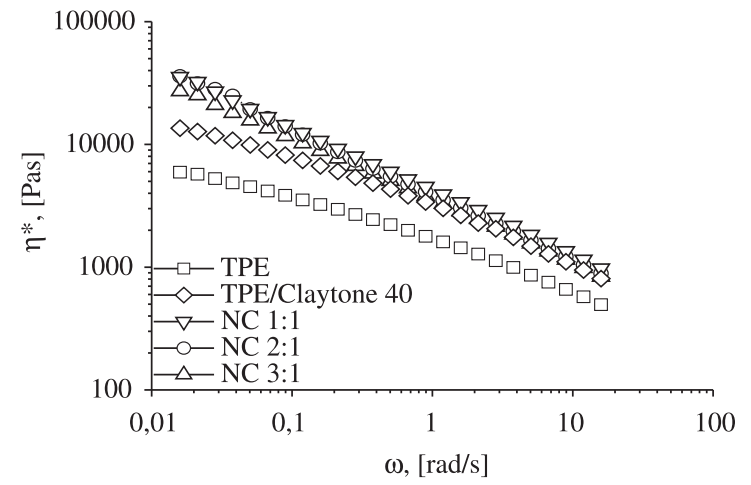

(b)

Figura 4. Módulo elástico (a) e viscosidade complexa (b) em função da freqüência angular.

Tabela 2. Propriedades mecânicas do TPE e dos nanocompósitos com 5\% de argila.

\begin{tabular}{lccc}
\hline \multicolumn{1}{c}{ Amostra } & $\begin{array}{c}\sigma^{*} \\
(\mathbf{M P a})\end{array}$ & $\begin{array}{c}\varepsilon^{*} \\
(\boldsymbol{\%})\end{array}$ & $\begin{array}{c}\text { E secante } \\
(\mathbf{M P a})\end{array}$ \\
\hline TPE & $13,8 \pm 0,8$ & $325 \pm 31$ & $677 \pm 54$ \\
TPE/Claytone 40 & $12,8 \pm 0,7$ & $439 \pm 25$ & $575 \pm 59$ \\
NC 1:1 & $11,3 \pm 0,9$ & $464 \pm 45$ & $859 \pm 78$ \\
NC 2:1 & $12,2 \pm 0,6$ & $448 \pm 28$ & $749 \pm 69$ \\
NC 3:1 & $12,2 \pm 0,5$ & $480 \pm 10$ & $849 \pm 91$ \\
\hline
\end{tabular}

classificado como pseudo-sólido e que segundo a literatura ${ }^{[1,12,13]}$ é típico de nanocompósitos com estrutura intercalada e/ou esfoliada. Tal característica encontra-se de acordo com os resultados obtidos na difração de raio X. Nota-se também que as curvas dos nanocompósitos com as diferentes razões PP-MA/Argila foram bastante próximas.

\section{Propriedades mecânicas}

As propriedades de tração do elastômero termoplástico (TPE) e dos nanocompósitos foram avaliadas com o intuito de verificar a influência das diferentes proporções dos concentrados (Tabela 2). Não houve variação significativa dos valores de tensão na ruptura $\left(\sigma^{*}\right)$, porém os valores de deformação na ruptura $\left(\varepsilon^{*}\right)$ aumentaram em função da adição da argila Claytone 40 e do agente interfacial PP-MA. Esta tendência pode ser atribuída a uma possível ação plastificante do surfactante presente na argila Claytone 40 e do PP-MA. Por outro lado, constatou-se aumento do módulo, ou seja, da rigidez dos nanocompósitos obtidos a partir da adição dos concentrados (PP-MA/Claytone 40) ao TPE. Este comportamento pode ser atribuído ao maior grau de dispersão da argila Claytone 40 na matriz de TPE, propiciado pela ação do agente interfacial (PP-MA), ressaltando que de acordo com as análises de DRX essas amostras apresentaram menor intensidade e maiores valores de $\mathrm{d}_{001}$, quando comparadas ao sistema sem PP-MA. É interessante notar que a adição direta da argila Claytone 40 ao TPE resultou em menor módulo, pode ser que o menor grau de dispersão desta argila na matriz, conforme demonstrado nas análises de DRX e do mapeamento de silício seja responsável pela redução da propriedade.

\section{Conclusão}

A obtenção de nanocompósitos de TPE e argila organofílica (Claytone 40) é dependente da presença de um terceiro componente que tenha grupos polares e seja compatível com a matriz polimérica, no caso o PP-MA. Outro fator importante é a razão entre agente interfacial e a argila organofílica, tendo em vista que há modificações no grau de cristalinidade e no processo de cristalização. Em última instância tais modificações em conjunto com o tipo de estrutura obtida, intercalada e/ou esfoliada, são responsáveis pelo desempenho mecânico do nanocompósito. Especificamente, em relação ao sistema estudado a razão PP-MA/Claytone 40 3:1 apresentou o melhor conjunto de resultados, preservando o grau de cristalinidade do TPE puro e melhorando as propriedades de tração.

\section{Referências Bibliográficas}

1. Deri, G.; Lafleur, P. G. \& Dubois, c. - Polym. Compos., 29, p.1301 (2008).

2. Barick, A. K. \& Tripathy, D. K. - J. Appl. Polym. Sci., 117, p.639 (2010). http://dx.doi.org/10.1002/app.31303

3. Moraes, R. P.; Valera, T.; Pereira, A. M. C.; Demarquette, N. R. \& Santos, A. M. - J. Appl. Polym. Sci., 119, p.3658 (2011). http://dx.doi. org/10.1002/app.33067

4. Santos, K. S.; Castel, C. D.; Liberman, S. A.; Oviedo, M. A. S. \& Mauler, R. S. - J. Appl. Polym. Sci., 119, p.1567 (2011). http://dx.doi. org/10.1002/app.32828

5. Nayak, S. K.; Mohanty, S. \& Samal, S. K. - J. Appl. Polym. Sci., 117, p.2101 (2010).

6. Metha, S.; Mirabella, F. M.; Ruefner, K. \& Bafna, A. - J. Appl. Polym. Sci., 92, p.928 (2004). http://dx.doi.org/10.1002/app.13693

7. Kim, D. H.; Fasulo, P. D.; Rodgers, W. R. \& Paul, D. L. - Polymer, 48, p.5960 (2007). 
8. Semenov, A. N. \& Rubinstein, M. - Macromolecules, 34, p.1058 (2001). http://dx.doi.org/10.1021/ma0013049

9. Reichert P.; Nizt H.; Klinke S.; Brandsch R.; Thomann, R. \& Mülhaupt, R. - Macromol. Mater. Eng., 275, p.8 (2000). http://dx.doi.org/10.1002/ (SICI)1439-2054(20000201)275:1<8::AID-MAME8>3.0.CO;2-6

10. Xu, G.; Chen, G.; Ma, Y.; Ke, Y. \& Han, M. - J. Appl. Polym. Sci., 108, p.1501 (2008). http://dx.doi.org/10.1002/app.27750
11. Hejazi, I.; Seyfi, J.; Sadeghi, G. M. M. \& Davachi, S. M. - Mater. Des., 32, p.649 (2011). http://dx.doi.org/10.1016/j.matdes.2010.08.009

12. Austin, J. R. \& Kontopoulou, M. - Polym. Eng. Sci., 46, p.1491 (2006). http://dx.doi.org/10.1002/pen.20622

13. Shu-Ying, G.; Ren, J. \& Qin-Feng, W. - J. Appl. Polym. Sci., 91, p.2427 (2004).

Enviado: 30/06/10

Reenviado: $25 / 07 / 11$

Aceito: 02/09/11 